\title{
On the Influence of Nozzle-generated Coherent Structures on the Primary Atomization of Fuel Jets
}

\author{
Jerome Hèlie ${ }^{1}$, Federico Piscaglia*2 \\ ${ }^{1}$ Advanced System Engineeing, VItesco Technologies, Toulouse, France \\ ${ }^{2}$ Department of Aerospace Science and Technology (DAER), Politecnico di Milano, Italy \\ *Corresponding author email: federico.piscaglia@polimi.it
}

\begin{abstract}
The application of a 3-phase VOF-LES solver [1] to the simulation of two transparent nozzle replicas is presented. The solver, implemented in the OpenFOAM Technology, is able to capture in-nozzle cavitation and fuel atomization near the nozzle exit. Simulations show that flow features are influenced by the sac and the hole geometry. It is found that the primary flow deviates in the sac, and that a secondary swirling flow generates. Depending on their importance, counter rotating vortices generates in proximity of the hole entrance. When the fluid pressure is about the saturation pressure, the flow cavitates and vortex strings are generated in newly formed vortex cores. Detailed flow unsteadiness and vortex pair instabilities change the instantaneous flow topology. No hydraulic flip is found in the tested real-size conical hole geometries. The nozzle flow features can be therefore related to the external flow results. The present work focuses on so-named "primary atomization" which is in fact related to cavitation collapse, liquid dispersion and interface wrinkling and breakup.
\end{abstract}

\section{Keywords}

Atomization, cavitation, LES-VOF, coherent structures, OpenFOAM.

\section{Introduction}

There are many applications of the spray theory related to aeronautics, power, propulsion, heat transfer and materials processing. The flow inside the nozzle, guided by the injector design, has a dominant effect on the generation of spray patterns and propellant-air mixing $[1,2]$. This is especially true in the presence of cavitation, where the flow field inside the nozzle must be regulated to maximize the available liquid surface area at the nozzle exit to facilitate mass, momentum and heat transfer. A characterization of the flow inside the nozzle is difficult, both experimentally and numerically $[3,4,5]$. Numerical simulations require robust numerical schemes and models to accurately capture the turbulent, multiphase nature of the flow (liquid fuel, gaseous fuel and air) and large computational resources are needed to capture the sharp liquid/vapor interfaces on high-resolution grids. Simplified realistic size two-dimensional holes were initially considered in [6] and are still useful for basic simulation model validation or wellcontrolled optical measurements [7, 8]. However, keeping the nominal geometry or managing a complex shape when considering real-size nozzles and high-pressure conditions becomes very difficult. A proof of concept of a real-size, real-shape, real-pressure, representative surface roughness, transparent nozzles for Design of Experiments [9, 2], is used to validate a LESVOF solver developed by the authors in [1]. The aim of this work is to present results of a study, including both experiments and simulations, of the flow characteristics in high pressure liquid injection of $n$-Heptane at a pressure of 100 bar. Two different configurations of realsize transparent glass-nozzle replicas of injectors were built by Vitesco Technologies for this study, using a novel rapid prototyping technique based on laser-etched manufacturing and presented in [2, 9]. High-resolution simulations of the injector flows were conducted using an in-house Finite Volume (FV) variable-density multiphase VOF solver developed by the authors 
[1] in the OpenFOAM Technology, that is included in a in-house $\mathrm{C}++$ library developed by the authors. Validated results from the simulations were post-processed to investigate the physical mechanism leading to the flow instability inside the nozzle.

\section{Experimental setup}

Ad-hoc prototypes of real-sized glass nozzle injectors used for this study are capable of operating under realistic nozzle pressures; they were designed and built by Vitesco Technologies to contribute to the nozzle design and cavitation control efforts aimed at improving high-pressure fuel injectors [9]. The geometrical features of the prototype have been thought to reproduce a single-hole injector, representing a $60^{\circ}$ sector of a real multi-hole injector, as reported in Fig. 1. This includes the needle, the sac geometry, the hole geometry and the external nozzle shape.
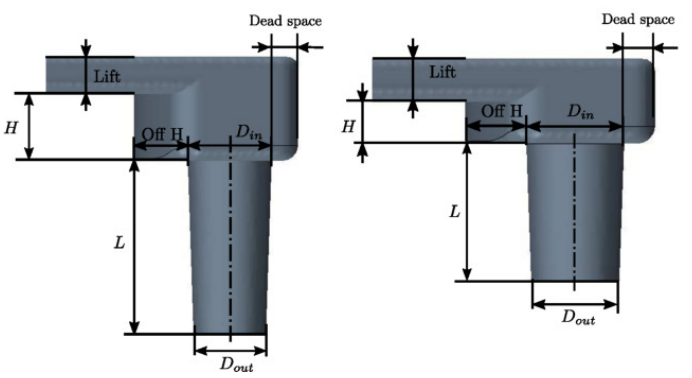

\begin{tabular}{|l|c|c|}
\hline \multirow{2}{*}{} & \multicolumn{2}{|c|}{ Length $[\mu \mathrm{m}]$} \\
\cline { 2 - 3 } lift & $\mathrm{ID}-3$ & $\mathrm{ID}-10$ \\
nozzle length $(\mathrm{L})$ & 351 & 79 \\
sac height $(\mathrm{H})$ & 136 & 241 \\
hole offset $($ off-H) & 102 & 78.5 \\
inlet diameter $\left(D_{\text {in }}\right)$ & 166 & 78.5 \\
outlet diameter $\left(D_{\text {out }}\right)$ & 147 & 148 \\
dead space length & 38 & 44.9 \\
\hline$K_{\text {geom }}=\left(\frac{D_{\text {in }}-D_{\text {out }}}{L}\right) \cdot 100$ & 12.92 & 10.52 \\
\hline
\end{tabular}

Figure 1. The nozzle geometries studied, named as configuration ID-3 (left), ID-10 (right). Dimensions are specified in the Table.

The injector prototype has been built in quartz glass (fused silica) by a laser-etched manufacturing process that is able to achieve an average surface roughness of $1-3 \mu \mathrm{m}$. Design values are listed in the Table of Fig. 1, while main features are shown in Fig. 2.

\begin{tabular}{lccc}
\hline \hline & \multicolumn{3}{c}{ Fluid } \\
\hline & $\mathrm{C}_{7} \mathrm{H}_{16, \text { liq }}$ & $\mathrm{C}_{7} \mathrm{H}_{16, \text { vap }}$ & air \\
\hline density $\rho_{l}\left[\mathrm{kgm}^{-3}\right]$ & 684 & 4.25 & 1.225 \\
dynamic viscosity $\mu_{l}\left[\mathrm{~kg} \mathrm{~m}^{-1} \mathrm{~s}^{-1}\right]$ & $4.0835 \times 10^{-4}$ & $7.0125 \times 10^{-6}$ & $1.7885 \times 10^{-5}$ \\
surface tension $\left[\mathrm{Nm}^{-1}\right]$ & 0.019517 & - & - \\
saturation pressure $[\mathrm{Pa}]$ & 7000 & - & - \\
\hline
\end{tabular}

Table 1. Thermodynamic properties for $\mathrm{n}$-Heptane $\left(\mathrm{C}_{7} \mathrm{H}_{16}\right): \mathrm{C}_{7} \mathrm{H}_{16}$,liq, $\mathrm{C}_{7} \mathrm{H}_{16 \text {,vap }}$ at $\mathrm{T}=25^{\circ} \mathrm{C}$, and non-condensable gas (air) at $\mathrm{T}=15^{\circ} \mathrm{C}$

Experiments have been carried out at Vitesco Technologies at the facility in Toulouse (France). The aim of the experiments was to provide a detailed visualization of the flow within the nozzle and the extent of the primary jet breakup. Experimental tests of liquid-into-air injection have been carried out at a needle lift of $79 \mu \mathrm{m}$, that is representative of a fully opened nozzle using $\mathrm{n}$ Heptane as the working fluid (thermophysical and transport properties of $n$-Heptane are listed in Tab. 1). Both the numerical solver and the test bench can work with real fuels and are not limited to $\mathrm{n}$-Heptane. Tests have been carried out using a camera for two different viewing angles, $\theta_{1}=0^{\circ}$ (front view) and $\theta_{2}=90^{\circ}$ (lateral view), $\theta$ being the azimuthal angle. The average jet penetration over twenty experiments of ten different geometrical configurations (ID-1 to ID-10) was measured. Configurations ID-3 and ID-10 (see Fig. 1) showed respectively the maximum and minimum penetrations in the injection time interval $(1400 \mu s)$ tested; these configurations were hence chosen for numerical validation and analysis. They have identical lift and similar 
values of nozzle inlet and outlet diameters. They are designed to deliver similar mass flow rate, but with different: a) nozzle sac geometry, through the height of the step $\mathrm{H}$, the hole offset (off$\mathrm{H})$ and the length of the dead space; b) nozzle length. They operate at the conditions reported in Tab. 2.

\begin{tabular}{cccc}
\hline \hline Parameter & ID-10 & ID-3 & Unit \\
\hline pinlet & 100 & 100 & bar \\
pamb & 1 & 1 & bar \\
Mean liquid Velocity $U_{l}$ & 155.07 & 149.43 & $\mathrm{~m} \mathrm{~s}^{-1}$ \\
Re at plane $N_{6}$ & 36110 & 35104 & - \\
Re at plane $S_{1}$ & 14499 & 13020 & - \\
$K$ & 1.0095 & 1.0095 & - \\
$C$ & 104.9422 & 104.9422 & - \\
We & 110049 & 104712 & - \\
\hline
\end{tabular}

Table 2. Operating conditions of the experimental tests applied to nozzle configurations ID-3 and ID-10 at the nozzle exit [2].

The inlet fuel pressure has been set to 100 bar. The outlet of the nozzle is ambient temperature and pressure. While the solver assumes isothermal conditions of the working fluid, Tab. 1 reports the fluid properties computed at two different temperatures. In experiments usually nHeptane and air have different temperatures: n-Heptane is usually warmer, at $\approx 25^{\circ} \mathrm{C}$, while air in the injection chamber stays at $\approx 15^{\circ} \mathrm{C}$. Under the isothermal approximation, the sensible heat exchange that would have been occurred during fuel-air mixing is neglected.

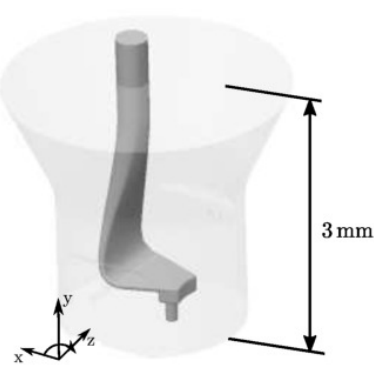

(a)

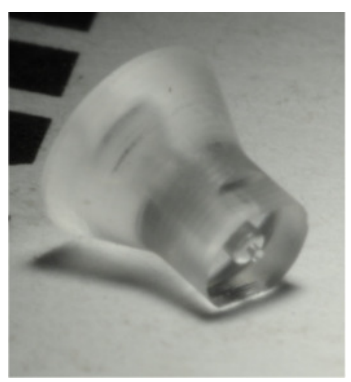

(b)

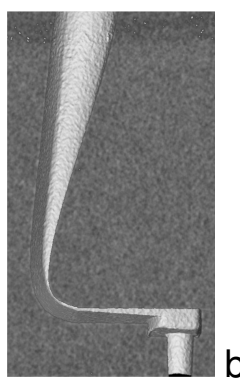

(c)

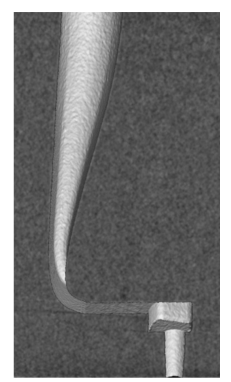

(d)

Figure 2. Sample of transparent glass nozzle replica: a) external view; b) sample of a central-nozzle hole; c) XCT visualization of glass nozzle side of ID-3; d) XCT visualization of glass nozzle side of ID-10.

\section{Computational Methodology for the Simulation of Cavitation in High-Pressure Injectors}

CFD simulations have been performed on the XCT-based virtual model of the real component, rather than on the ideal CAD geometry. The workflow of the combined XCT-CFD procedure is summarized in Fig. 3. It is important to mention that the effect of the surface roughness is not accounted in the CFD simulations: despite the grid used is quite fine ( $80 \mathrm{M}$ cells), it is still not fine enough to account for the influence of the roughness on the evolution of the boundary layers on the walls at the Reynolds numbers studied. It is worth mentioning that high resolution XCT cannot properly capture sharp edges; this may be a serious limit when dealing with injectors, because sharpness of the edges strongly influences the development of cavitation at the nozzle entrance. To correct this bias, measurements using a microscope were therefore used to quantify the hole edge rounding and to verify the average wall roughness (that were both found to be about $1 \mu \mathrm{m}$ ); in particular, some preliminary representative samples of injectors were broken to perform optical measurement of the hole entrance. Manual corrections were therefore applied to the CAD file in proximity of the sharp corners at the nozzle entrance, where information from the microscope were available. The grid consists of 80 million of polyhedral 
cells, that allow different refinement levels in the different regions of the injector geometry, as shown in Fig. 3. LES turbulence modeling [10] has been used for the simulations, despite of its high computational cost, because of its better ability to characterize the different flow scales and therefore to capture the cavitation onset in the nozzle [11,12]. A detailed description about the theory of the three-phase variable-density multiphase VOF solver developed by the authors used in this work and its verification can be found in [1], while validation on complex nozzle geometries can be found in [2].
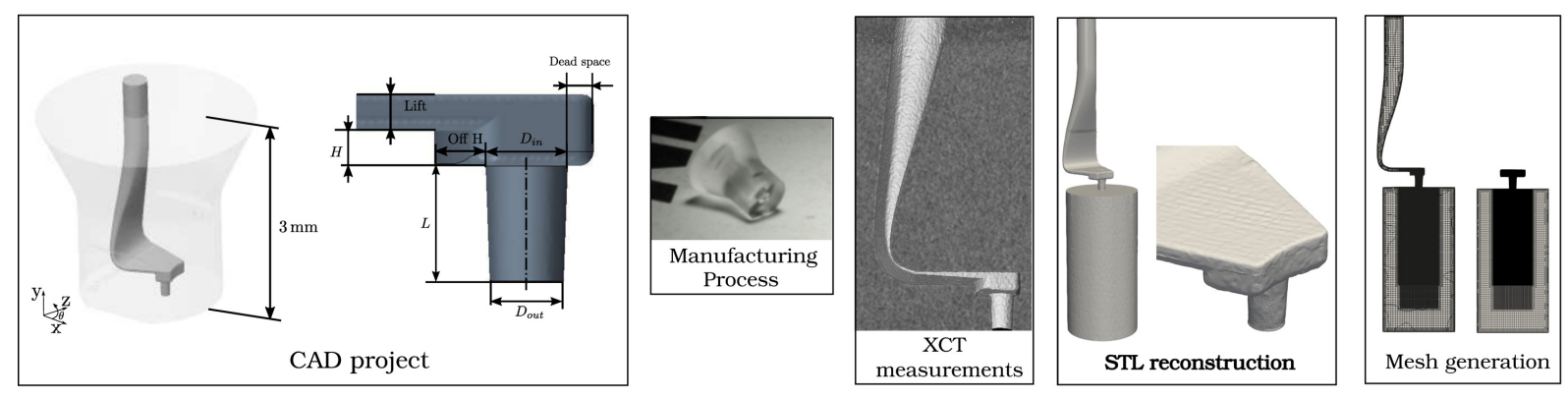

Figure 3. Sketch of the workflow for the generation XCT-based virtual model of the injectors studied. Since high resolution XCT cannot properly capture sharp edges, measurements from the microscope were used to quantify the hole edge rounding at the nozzle entrance. Manual corrections in the STL file, before the automatic generation of the polyhedral mesh.

\section{Validation of the Computational Methodology}

A validation by the comparison with experiments is presented, to prove the reliability of the solver to describe the cavitating structures occurring in the real glass nozzle injector. Experimental shadowgraph pictures of the cavitating areas have been realized using a long-range microscope on the glass nozzle. A half-inch flexible fiber optic light guide mated with a powerful LED illuminator (white steady light). Bubbles appear dark in the image, while liquid regions appear bright. Simulations results are generated as projected views of the three-dimensional iso-surfaces $\left(\alpha_{v}=0.3\right)$ of the fuel-vapor. We report a comparison of numerical and experimental results on the front $\left(\theta=0^{\circ}\right)$ and lateral (side) view $\left(\theta=90^{\circ}\right)$ for the two injector configurations studied in this work. This kind of analysis can give a measure of reliability of the solver in describing the cavitating structures occurring inside the nozzle. Being the sharpness of the edge at the hole entrance directly linked to the intensity of the cavitation, the sharp edges at the hole entrance in the XCT-based virtual model have been manipulated by a CAD software; this ensures to be coherent with the measurements at the microscope and to cancel out the possible errors coming from the XCT-based reconstruction of the virtual model on those regions (see Fig. 3). The geometry has also been directly measured to generate the $\mathrm{CAD}$, capturing some discrepancy in the asymmetry of the hole entrance. In Figs. 4 and 5, a selection of instantaneous events from simulations and experiments are compared. The experimental visualizations using the front and lateral view are helpful to understand the contribution of each type of cavitation and the multiphase flow topology of each zone. In the cases studied, a moderate shear cavitation appears quite rarely, randomly, and it often involves a small portion of the nozzle area. Shear cavitation is directly driven by the low pressure area in the detached flow region in proximity of the hole entrance. Few aspects help to explain this moderate shear cavitation in the two injector configurations. The geometry is a sector of a complex real nozzle: the shape includes a confined, non-negligible sac area. The role of the initial steep transition from the intake pipe to the sac volume causes a strong flow recirculation in the sac. 


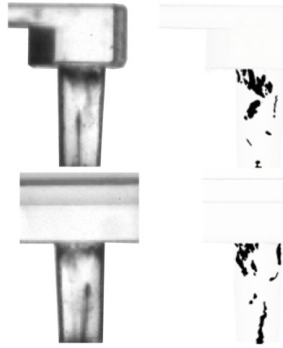

(a)

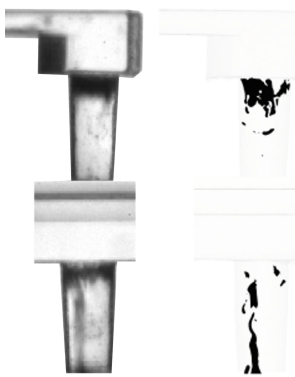

(d)

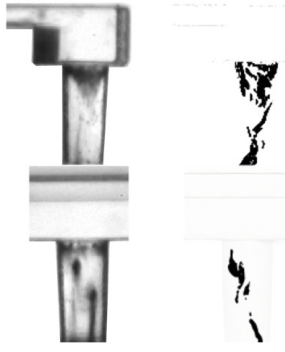

(b)
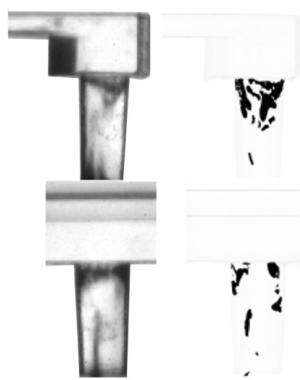

(e)

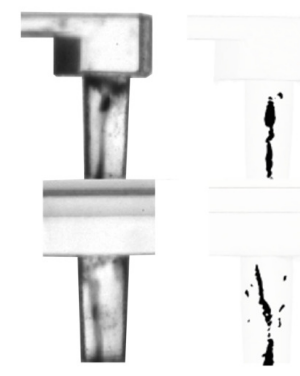

(c)

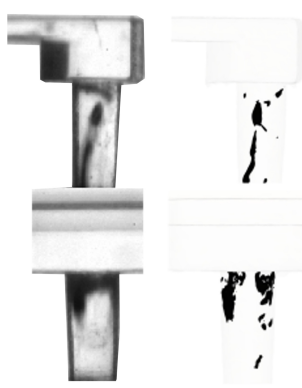

(f)

Figure 4. Configuration ID-3. Comparison of detected cavitation structures from experimental instantaneous shadowgraphies (left) and simulations (right). Rows 1, 3: lateral view $\left(\theta=90^{\circ}\right)$. Rows 2, 4: front view $\left(\theta=0^{\circ}\right)$.

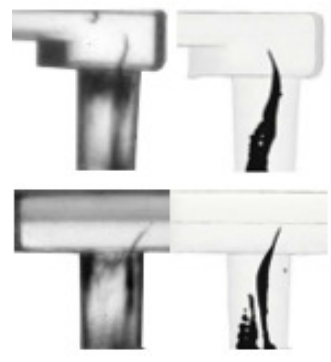

(a)

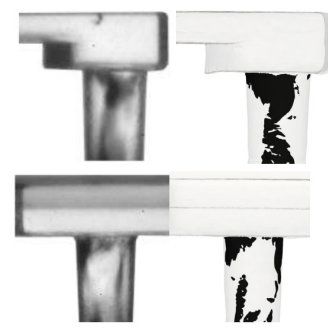

(d)

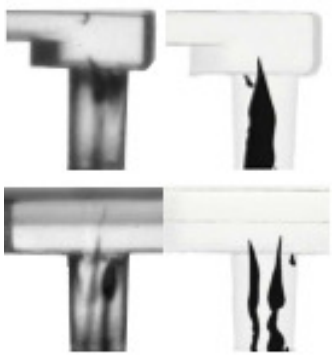

(b)

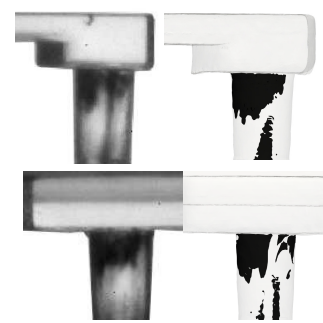

(e)

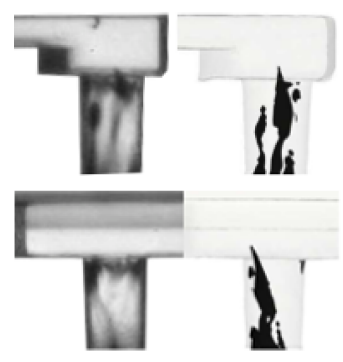

(c)

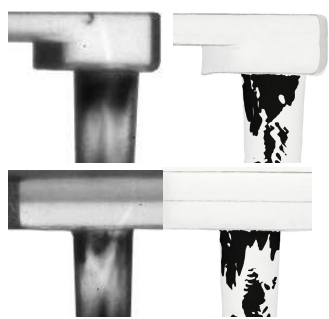

(f)

Figure 5. Configuration ID-10. Comparison of detected cavitation structures from experimental instantaneous shadowgraphies (left) and simulations (right). Rows 1, 3: lateral view $\left(\theta=90^{\circ}\right)$. Rows 2, 4: front view $\left(\theta=0^{\circ}\right)$. 
Lastly, shear cavitation cannot develop along the complete hole due to its conicity and it vanishes at almost one third of the hole. Injectors ID-3 and ID-10 show very similar behavior in terms of shear cavitation. With such a moderate development of shear cavitation, the position of the strings of fuel-vapor can be quite easily distinguished by the shadowgraph pictures. This is especially useful because a large amount of string cavitation develops in the nozzle. String cavitation appears visually as vertical filaments. They can appear as a single full ligament, covering all the hole length. They can also appear partly at the hole entrance, at the hole end or in both (see Fig. 4 and 5). Ligaments mostly appear as vertical strings but some slight bending can be visible. String cavitation is more visible than shear cavitation in the geometries studied. This is a surprising result that will be discussed in detail in the next sections. Additionally, in configuration ID-10, string cavitation is more intense than in ID-3. Time evolutions of cavitation and velocity distribution are discussed in [2].

(a) sac cutting-plane

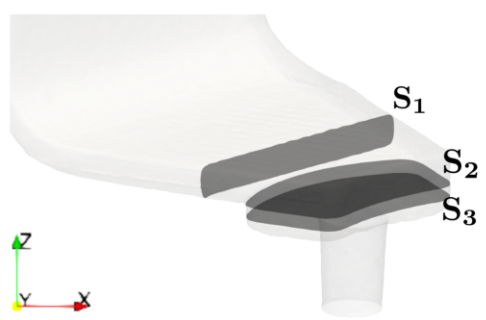

(b) nozzle cutting-plane

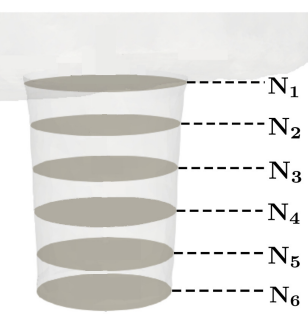

(c) domain cutting-plane

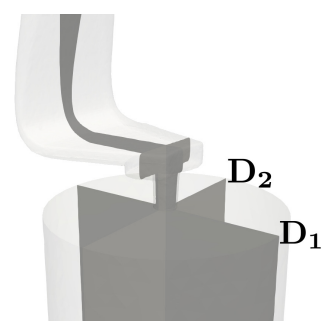

Figure 6. The flow analysis is based on time- and space- averaged flow quantities over: a) the three cross sections $\mathbf{S}_{1}, \mathbf{S}_{2}$ and $\mathbf{S}_{3}$ located in the sac; b) cross-planes $\mathbf{N}_{\mathbf{i}}, \mathbf{i} \in[1 ; 6]$ along the nozzle; c) two cutting planes, $\mathbf{D}_{\mathbf{1}}$ (lateral) and $\mathbf{D}_{2}$ (frontal), parallel to the nozzle axis are defined by the $\theta_{1}=0^{\circ}$ and $\theta_{2}=90^{\circ}$ respectively.

\section{Flow Analysis}

A main goal of this research is to identify the mechanisms driving to very different flow atomization in the two injector nozzles. The two injectors studied operate at the same injection pressure and very similar Mass Flow Rate (MFR). As discussed in [2], the volume of the region where flow recirculation occurs is not very different among the two geometries. Also the sector angle is the same. They present different sac heights and hole-to-step distances (named here offs$\mathrm{H}$ ) though, while the fuel pattern in the injector sac volume does not show relevant differences in the flow features. Also the flow pattern at the hole entrance is very similar.

A comparison between the two injectors has been carried out by looking at the main features of the flow in the nozzle holes (see Fig. 7a). Quantities over six equispaced parallel planes in the nozzle holes $\left(\mathbf{N}_{1}-\mathbf{N}_{6}\right.$ of Fig. 6$)$ are plotted. The position of each plane is normalized by the length of the nozzle. From Figs. 7a, it is apparent that the axial component of the flow velocity is progressively increasing over the injector nozzles. This is a direct consequence of the combined effect of the nozzle conicity $K_{g}$ and of the cavitation. Swirling vortices generated at the nozzle entrance expand and progressively disappear in proximity of the nozzle end (planes $\mathbf{N}_{4}$ $\mathbf{N}_{6}$ ); the magnitude of the non-axial velocity therefore reduces (Fig. $7 \mathrm{~b}$ ) and the axial velocity progressively increases (Fig. 7a), so that the ratio among the two quantities is decreasing (Fig. 7c). Because of the acceleration of the flow in the hole (Fig. 7a), shear cavitation tends to disappear. The larger non-axial velocity in injector ID-10 (see Fig. 7b) favors a stronger string cavitation. This is also confirmed by Fig. 7f. The corresponding time-averaged mean pressure over planes $\mathbf{N}_{1}-\mathbf{N}_{6}$ is shown in Fig. $7 \mathrm{~d}$. As expected, time-averaged pressures on planes $\mathbf{N}_{1}$ and $\mathbf{N}_{6}$ are the same, being forced by the boundary conditions. A stronger pressure recovery to the atmospheric pressure is noticed for ID-10 near the nozzle exit, between planes $\mathrm{N}_{5}$ and $\mathbf{N}_{6}$. The larger conicity factor $k_{\text {geom }}$ of injector ID-3 favors a pressure recovery of almost the $85 \%$ of the nozzle average inlet pressure, with a local maximum at plane $N_{3}$. The longer hole of 
injector ID-3 allows for the formation of larger detachment regions at the nozzle entrance and favors a different intensity of swirl cavitation. The vorticity magnitude (Fig. 7e) has a similar trend in the two configurations, but ID-3 shows a stronger decay while the flow is reaching the nozzle end. Also, the liquid area of the flow on the different nozzle sections $\mathbf{N}_{1}-\mathbf{N}_{6}$ (Fig. $7 f$ ) is larger for injector ID-3, as a consequence of a weaker cavitation of the liquid along the nozzle. The higher conicity of injector ID-3 results in a lower atomization of the liquid, that fills most of the nozzle area on sections $\mathbf{N}_{3}-\mathbf{N}_{6}$.

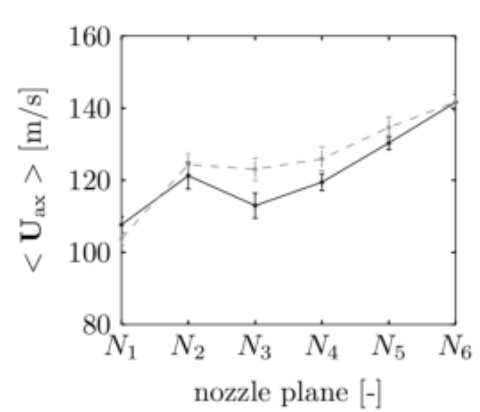

(a)

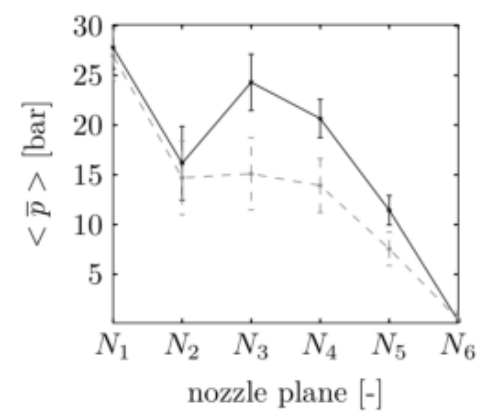

(d)

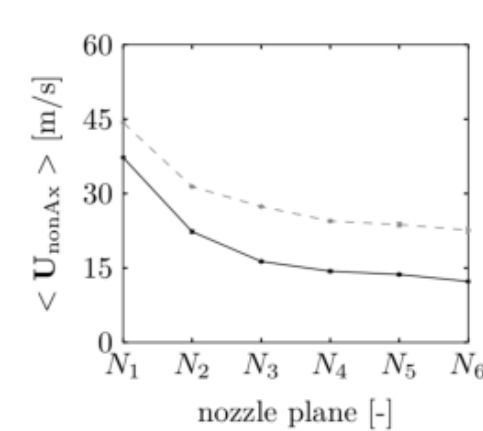

(b)

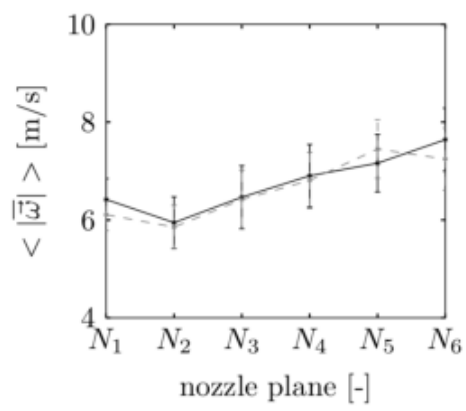

(e)

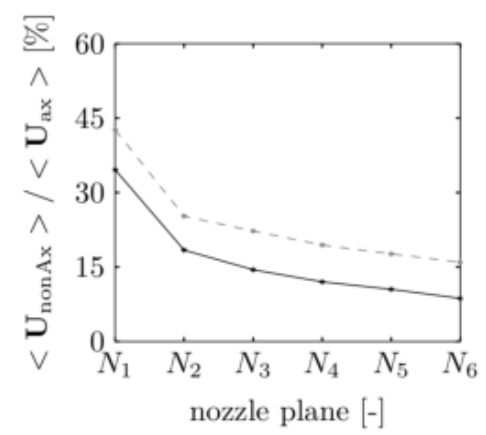

(c)

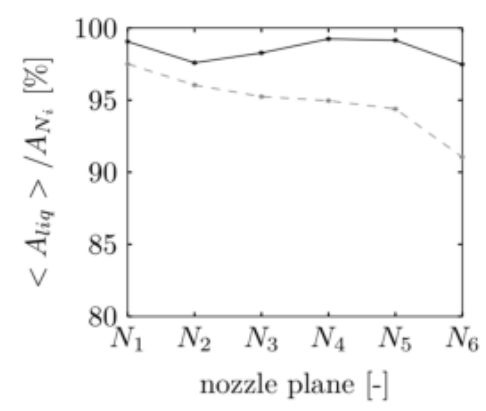

(f)

Figure 7. Mean values over the nozzle axis, on planes $N_{1}$ to $N_{6}$, of time-averaged quantities: 1) MFR-weighted average axial velocity; b) magnitude of the MFR-weighted average non-axial velocity; c) radial/axial velocity ratio; d) average pressure; e) MFR-weighted vorticity magnitude; f) liquid area over cross sectional area. Legend: - ID-3; - ID-10.

\section{Conclusions}

A three-phase LES solver [1] has been used to carry out a study about the evolution of string cavitation on two transparent nozzle replicas of real high pressure injectors, namely configurations ID-3 and ID-10. Both geometries represent one sixth of a sector with a multi-hole injector. The different performance of the two injectors is due to their geometrical features (e.g nozzle diameters, height, dead spaces) and to the inlet flow conditions developing from the small details in the sac. The agreement between simulations and experimental visualizations of the cavitating flow observed by two views (frontal and side) is satisfying. Validated results have been then used to analyze the nozzle flow fields. It s shown that non-axial kinetic energy in the nozzle seems to be a relevant parameter to characterize the motion of large structures and the intensity of the in-nozzle cavitation. On the other hand, no conclusive remarks to optimize the injector design can be still drawn: the size and distribution of the maximum ligaments out of the nozzle exit and the spatial dispersion/homogeneity of the spray in the outer region are some of the additional relevant parameters that must also be taken into account. These parameter are related to the study of the primary atomization, that will be the topic of upcoming publications. 


\section{Acknowledgements}

Authors gratefully acknowledge the Laboratory Computing Resource Center (LCRC) at Argonne National Laboratory for the computing resources provided through the KNL-OPENFOAM-VOF project.

\section{References}

[1] F. Giussani, F. Piscaglia, G. Saez-Mischlich, and J. Hèlie, "A three-phase VOF solver for the simulation of in-nozzle cavitation effects on liquid atomization," Journal of Computational Physics, vol. 406, p. 109068, 2020.

[2] F. Piscaglia, F. Giussani, J. Hèlie, N. Lamarque, and S. Aithal, "Vortex Flow and Cavitation in Liquid Injection: A Comparison between High-Fidelity CFD Simulations and Experimental Visualizations on Transparent Nozzle Replicas," International Journal of Multiphase Flow, vol. 138, p. 103605, 2021.

[3] R. Torelli, S. Som, Y. Pei, Y. Zhang, and M. Traver, "Influence of fuel properties on internal nozzle flow development in a multi-hole diesel injector," Fuel, vol. 204, pp. 171 - 184, 2017.

[4] F. Piscaglia, F. Giussani, A. Montorfano, J. Hélie, and S. Aithal, "A multiphase dynamic-vof solver to model primary jet atomization and cavitation inside high-pressure fuel injectors in openfoam," Acta Astronautica, 2018.

[5] Hannebique, G. and Sierra, P. and Riber, E. et al., "Large Eddy Simulation of Reactive TwoPhase Flow in an Aeronautical Multipoint Burner." Flow Turbulence Combustion, vol. 90, p. 449-469, 2013.

[6] E. Winklhofer, E. Kull, E. Kelz, and A. Morozov, "Comprehensive hydraulic and flow field documentation in model throttle experiments under cavitation conditions," in ILASS 2001, 17th European Conference on Liquid Atomization and Spray Systems, Zurich, Switzerland, 2001.

[7] C. Mauger, L. Méès, M. Michard, A. Azouzi, and S. Valette, "Shadowgraph, Schlieren and interferometry in a 2D cavitating channel flow," Experiments in Fluids, vol. 53, no. 6, pp. 1895-1913, 2012. [Online]. Available: https://hal.archives-ouvertes.fr/hal-00780132

[8] W. H. Nurick, "Orifice cavitation and its effect on spray mixing," Journal of Fluids Engineering, vol. 98, no. 4, p. 681, 1976. [Online]. Available: https: //doi.org/10.1115/1.3448452

[9] A. Agresta, J. Hélie, E. Kull, N. Lamarque, A. Lyubar, and S. Schuster, "Real-Size RealShape Real-Pressure Transparent Nozzles to Contribute to Nozzle Design and Cavitation Control for GDI," in 29th Conference on Liquid Atomization and Spray Systems, ILASSEurope, 2-4 September 2019, Paris, France.

[10] F. Nicoud and F. Ducros, "Subgrid-Scale Stress Modelling Based on the Square of the Velocity Gradient Tensor," Flow, Turbulence and Combustion, vol. 62, pp. pp. 183-200, 1999.

[11] M. Gavaises, F. Villa, P. Koukouvinis, M. Marengo, and J.-P. Franc, "Visualisation and les simulation of cavitation cloud formation and collapse in an axisymmetric geometry," International Journal of Multiphase Flow, vol. 68, pp. 14 - 26, 2015.

[12] R. E. Bensow and G. Bark, "Implicit LES predictions of the cavitating flow on a propeller," Journal of Fluids Engineering, vol. 132, no. 4, p. 041302, 2010. [Online]. Available: https://doi.org/10.1115/1.4001342 